\title{
PREDICTIVE MODEL FOR LIKELIHOOD OF DETECTING CHRONIC KIDNEY FAILURE AND DISEASE USING FUZZY LOGIC
}

\author{
Ajinaja Micheal $^{1}$ and Ajinaja Olayinka ${ }^{2}$ \\ ${ }^{1}$ Department of Computer Science, Federal Polytechnic, Ile - Oluji, Nigeria \\ ${ }^{2}$ Department of Electrical and Electronic Engineering, ObafemiAwolowo University, \\ Ile-Ife, Nigeria
}

\begin{abstract}
Fuzzy logic is highly appropriate and valid basis for developing knowledge-based systems in medicine for different tasks and it has been known to produce highly accurate results. Examples of such tasks include syndrome differentiation, likelihood survival for sickle cell anaemia among paediatric patients, diagnosis and optimal selection of medical treatments and real time monitoring of patients. For this paper, a Fuzzy logic-based system is untaken used to provide a comprehensive simulation of a prediction model for determining the likelihood of detecting Chronic Kidney failureldiseases in humans. The Fuzzy-based system uses a 4-tuple record comprising of the following test taken: Blood Urea Test, Urea Clearance Test, Creatinine Clearance test and Estimated Glomerular Filtrate rate(eGFR).Understanding of the test was elicited from a private hospital in Ibadan through the help of an experienced and qualified nurse which also follows same test according to National Kidney Foundation. This knowledge was then used in the developing the simulated and rule-base prediction model using MATLAB software. The paper also follows the 3 major stages of Fuzzy logic. The results of fuzzification of variables, inference, model testing and defuzzification of variables was also presented. This in turn simplifies the complication involved in detecting Chronic Kidney failure/disease using Fuzzy logic based model.
\end{abstract}

\section{KEYWORDS}

Fuzzy logic, prediction model, likelihood, chronic kidney diseaselfailure

\section{INTRODUCTION}

According to the National Kidney Foundation (2017) Chronic kidney disease (CKD) is a condition characterized by a gradual loss of kidney function over time and also known as chronic renal disease [1]. Chronic kidney disease also comprises environments that damage your kidneys and lessening their ability to keep you healthy by doing the jobs listed. If kidney disease gets poorer, wastes can build to high levels which in turn becomes extremely dangerous to the human body.

According to Dr EbunBamgboye (2013), President-elect of the Nephrology Association of Nigeria (NAN), 36.8 million Nigerians are suffering from kidney disease at different stages [2]. With this figure, it means that one in seven Nigerians is suffering from some form of kidney DOI: $10.5121 /$ ijcsitce.2018.5201 
The International Journal of Computational Science, Information Technology and Control Engineering (IJCSITCE) Vol.5, No.2, April 2018

disorder [2]. If not untested and untreated, complications can arise such as high blood pressure, anaemia (low blood count), weak bones, poor nutritional health and nerve damage [2]. He estimated about 15,000 new patients are diagnosed every year in the country. Unfortunately, only meagre number 1,000 patients can afford dialysis as at today among 50,000 patients who should ideally be on dialysis.

In Nigeria it is estimated that every year the incidence of kidney disease is 100 per $1,000,000$ population [3]. The number of new cases of kidney failure seen every year in the population of 170 million is 17,000. A study was carried out at the Kidney Care Centre, University of Medical Sciences, Ondo State by Oluseyi A Adejumo (2016) over an 18 month period and the following information were extracted from the patient's records: Socio-demographic data, referral hospital, mode of presentation, etiology of CKD, packed cell volume, blood pressure, and estimated glomerular filtration rate (GFR) at first presentation [4]. At the end of the study, the results of the research stated (among the 202 CKD patients with a male: female ratio of 1.7:1 and a mean age of $48.15 \pm 16.69$ years), the median estimated GFR of the patients at presentation was 3.17 $\mathrm{ml} / \mathrm{min} / 1.73 \mathrm{~m} \mathrm{[4]}$. The common etiologies of CKD were chronic glomerulonephritis, hypertension, diabetes mellitus, obstructive nephropathy in 69 (34.2\%), 47 (23.3\%), 38 (18.8\%), and $21(10.4 \%)$ respectively [4]. Among these patients, $111(55 \%)$ and $98(48.6 \%)$ had moderate to severe hypertension and anaemia, respectively, $173(85.6 \%)$ presented in CKD Stage 5, 101 $(50 \%)$ required urgent haemodialysis whereas 123 (60.9\%) required in-hospital admission. Only (18) $9 \%$ of these CKD patients presented by self-referral while (103) $51 \%$ were referred from secondary and private health facilities [4]. The presentation established that most Nigerian CKD patients still present very late to nephrologists indicating that the present precautionary strategies have not produced anticipated outcomes [4].

Fuzzy logic is a means of providing a path for the diagnosis and decision making process due to its ability to deal with uncertainties (fuzziness) and ambiguity which may exist in the knowledge and information relating to a domain of study [5]. In this paper, we would like to discuss how fuzzy logic can be used for developing an inference engine using CKD variables in simulation.

A fuzzy set is categorized via its membership function (MF). A membership function (MF) is a curve that defines how each point in the input space is mapped to a membership value (or degree of membership) between 0 and 1. The input space is sometimes referred to as the universe of discourse. Membership functions are used in the fuzzification and defuzzification steps of a FLS (fuzzy logic system), to map the non-fuzzy input values to fuzzy linguistic terms and vice versa [6].

The only condition a membership function must really satisfy is that it must vary between 0 and 1. The function itself can be an arbitrary curve whose shape we can define as a function that suits us from the point of view of simplicity, convenience, speed, and efficiency [7]. A classical set might be expressed as:

$$
A=\{x \mid x>n\}
$$

A fuzzy set is an extension of a classical set. If $\mathrm{X}$ is the universe of discourse and its elements are denoted by $\mathrm{x}$, then a fuzzy set $\mathrm{A}$ in $\mathrm{X}$ is defined as a set of ordered pairs.

$$
\mathrm{A}=\{\mathrm{x}, \mu \mathrm{A}(\mathrm{x}) \mid \mathrm{x} \in \mathrm{X}\}
$$


The International Journal of Computational Science, Information Technology and Control Engineering (IJCSITCE) Vol.5, No.2, April 2018

$\mu \mathrm{A}(\mathrm{x})$ is called the membership function (or MF) of $\mathrm{x}$ in $\mathrm{A}$. The membership function maps each element of $X$ to a membership value between 0 and 1. For this study, the following must be noted:

- Set A contains any input (CKD factors) or output (CKD likelihood) variable which will be considered for this study;

- Set $\mathrm{X}$ is provides the set of standards for which a variable is valid

- $\mu \mathrm{A}(\mathrm{x})$ is provides the map of the membership function that will be used to design the degree of membership

This paper is intended at developing a comprehensive fuzzy logic based system that predicts the likelihood of Chronic Kidney Disease/Failure. This is done by using a 4-tuple record which were gotten from a registered and experienced Doctor and Nurse (Nurse BunmiAdeyemo). Also according to the National Kidney Foundation (2014), there are several means of testing for CKD: Blood pressure, blood creatinine, blood urea test, urinalysis, urea clearance test, creatinine test, eGFR (estimated glomerular filtration rate) and renal ultrasound test. The choice of the 4 variables used were based on the expert advice from an experienced Doctor and Nurse. For this paper, Blood Urea test, eGFR (estimated glomerular filtration rate), Urea Clearance Test and creatinine clearance test.

\section{RELATED WORKS}

Farzad (2015) worked on Kidney Diseases Diagnosis by Using Fuzzy Logic. The paper aimed to develop a new method in fuzzy logic which can be used for kidney disease diagnosis [8]. An expressive study was carried out in Shafa kidney clinic in Tehran, Iran in 2012 [8]. The medical diagnosis fuzzy rules were formulated and applied using MATLAB software [8]. The fuzzy inference was applied to obtain a decision fuzzy set for each disease, and crisp decision values were attained to tell the certainty of existence for each disease. The results indicated that in the diagnosis of eight cases of kidney diseases through the examination of 21 indicators using fuzzy logic, kidney stone disease with $63 \%$ certainty was at the first level and renal tubular with $15 \%$ was at the lowest level and the other kidney diseases were at the other levels [8]. The prototype system checks for 8 suspected kidney infections and considers a total of 21 features.

Kerina et al (2017) worked on Risk Level Prediction of Chronic Kidney Disease Using NeuroFuzzy and Hierarchical Clustering Algorithm (s) [9]. Using designated topographies, prediction for CKD disease is done so as to recognize the risk. Using hierarchical clustering three clusters were formed to show that there is a solid connection amongst chronic kidney and diabetes. Using neuro-fuzzy system, predictions were done with a complete precision of $97 \%$. The effects of the prediction shows the risk of any patient having CKD given the ten features nominated from feature selection and were are clustered using hierarchical clustering. The 3 clusters formed recognise a group of patients who have higher risk of other related chronic diseases. In the paper, Cluster 1 showed that those in this cluster are similar in nature in that they have high haemoglobin, high sugar level, low blood urea, high packed cell, high red blood cell count, with pus cell clumps and without having diabetes mellitus form cluster 1 and these have CKD. Also, the majority in cluster 2 have sugar which is a little bit lesser than those in cluster 1 [9]. 
The International Journal of Computational Science, Information Technology and Control Engineering

(IJCSITCE) Vol.5, No.2, April 2018

Huong et al (2017) worked on using a simple questionnaire to detect chronic kidney disease patients. The study was limited to knowledge elicited from Long An province screening data in Vietnam. A cross sectional study was performed 2037 participants were recruited from 13 communes of Long An province, Vietnam, for CKD screening with urine albumin/creatinine ratio (ACR) measured by immuno-turbidimetric method and serum creatinine to estimate glomerular filtration rate (eGFR) [10]. CKD was defined as either ACR $\geq 30 \mathrm{mg} / \mathrm{g}$ or eGFR MDRD $<60$ $\mathrm{ml} / \mathrm{min} / 1.73 \mathrm{~m}^{2}$. A two page questionnaire with 23 variables was administered to each participant with queries postulated to be correlated with risk of CKD [10]. Of the 2037 participants, 260 $(12.76 \%)$ were found to have CKD. Five questionnaire variables (age more than 50, measured hypertension, history of diabetes, history of hypertension, and history of a low salt diet) were correlated with CKD, and used to construct a risk score for CKD [10].

\section{MATERIALS AND METHODS}

\section{a. Research Design}

In this paper, a fuzzy logic-based prediction model is planned with the goal of predicting the likelihood of chronic kidney disease (CKD). A detailed research design is presented in Figure 1 below. The design started with the identification of problem of predicting the likelihood of chronic kidney disease (CKD) using a number factors (tests) which will be considered as inputs (4 in all). Also, a detailed review of related literature was accessed to critically understand chronic kidney disease (CKD) and its symptoms coupled with the necessary tests used. Variables needed for the methodology was also sourced for and presented. Knowledge was elicited from a qualified and experienced Nurse at a privately Owned hospital in Ibadan, Oyo State, Nigeria. This was done in order to verify and properly understand all the information concerning CKD.

The knowledge gotten from the medical team was used in developing the inference engine for the proposed system which includes fuzzification of inputs and output variables, developing of model through the identification of aggregation and defuzzification for producing output variable which is the likelihood of CKD (Yes and No).

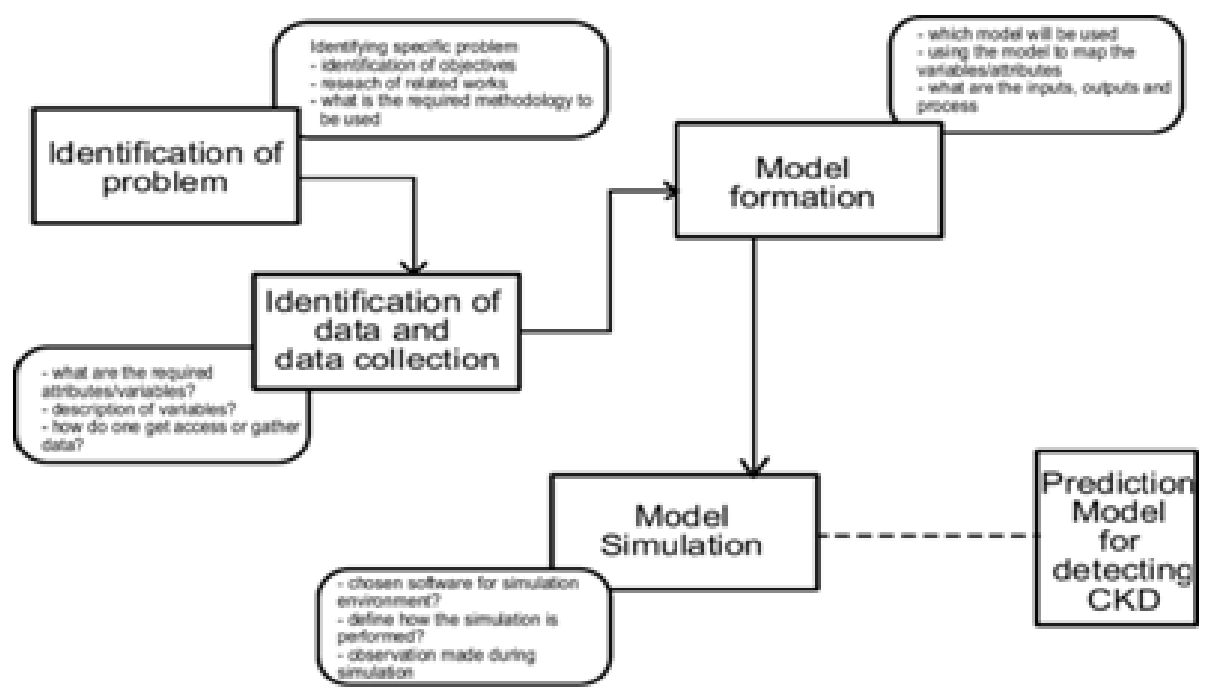

Figure 1: Research design for the study 
The International Journal of Computational Science, Information Technology and Control Engineering (IJCSITCE) Vol.5, No.2, April 2018

\section{b. Data identification and collection}

Often medical diagnosis involves the cautious examination of a patient to examine the presence and strength of some symptoms connected to a suspected illness in order to make a conclusion whether the patient suffers from that illness or not. Quite a number of symptoms/tests/factors are done in order ascertain whether or not a patient has Chronic Kidney Disease (CKD), among all these numerous tests which were considered for this study only 4 were considered as being the most significant: blood urea test, eGFR (estimated glomerular filtration rate), Urea clearance test and Creatinine clearance test. This information was gathered through an intensive and well patterned and organized meeting with an experienced and qualified Nurse and Doctor who were of help in identifying important tests for CKD likelihood. The blood urea test is defined as either: less than $20 \%, 20 \%$ less than and equal to Test less than and equal to $40 \%$, and greater than $40 \%$; eGFR is defined as either: less than $0.9 \%, 0.9 \%$ less than and equal to Test less than and equal to $1.2 \%$, and greater than 1.2\%; Urea Clearance test is defined as; less than $64 \%, 64 \%$ less than and equal to Test less than and equal to $99 \%$, and greater than $99 \%$; Creatinine clearance test is defined as; less than $0.93 \%, 0.93 \%$ less than and equal to Test less than and equal to $1.33 \%$, and greater than $1.33 \%$. The number of rules for required by the fuzzy logic inference system 32 $(4 * 4 * 2)$. This was done by multiplying the labels of each variable with each other.

\section{c. Fuzzy logic model formulation}

Considering the set of considered tests $\mathrm{T}$, expert physician's experience is obtained which can then be used in a fuzzy logic systems. To achieve this fuzzy logic system for this study, there are a number of events to be accomplished. The Fuzzy Logic System available in the Fuzzy Logic Toolbox of the MATLAB R2015a software has three parts (see Figure 2):

- A set of Inputs represented by their respective membership functions [11];

- An Inference Engine which contains the IF-THEN rules (domain knowledge) [11]; and

- An Output represented by its membership functions [11].

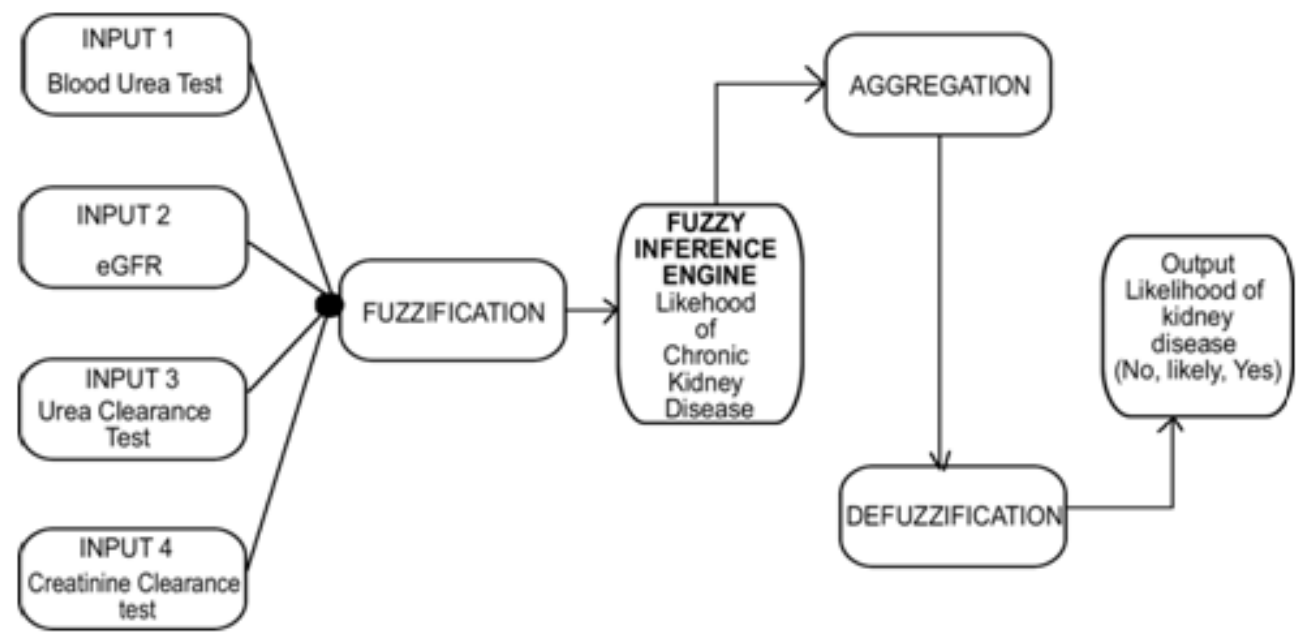

Figure 2 - Graphic diagram of the proposed model 
The International Journal of Computational Science, Information Technology and Control Engineering

(IJCSITCE) Vol.5, No.2, April 2018

The membership functions for the system will be used to map the values of each input and output variables into a $[0,1]$ interval with the use of mainly trapezoidal membership functions based on the pattern range distribution. This process is of fuzzification for the study. Immediately after fuzzification comes mapping of the fuzzified inputs. The fuzzified inputs are then mapped to the fuzzified output through AND, OR and NOT to develop IF-THEN rules. These rules describes the connection between all input (CKD factors) and output (likelihood of the chronic disease). The model will consist of different rules which are used to generate different results which are then aggregated to just one fuzzified output. This fuzzified output will then be defuzzified by the centroid method which chooses the centre of the polygon to define the label of the output variable as Yes, Likely or No [11].

In summary, the process of design and development of the fuzzy inference system needed for the prediction of Chronic Kidney Disease is as follows:

- Fuzzification of inputs and outputs;

- Construction of the inference engine;

- Rule aggregation; and

- Defuzzification of output variables.

\section{d. Defining membership functions}

A membership function (MF) is a curve that defines how each point in the input space is mapped to a membership value (or degree of membership) between 0 and 1 . The input space is sometimes referred to as the universe of discourse. A fuzzy set is completely characterized by its membership function (MF). In advance to the process of Fuzzification, all crisp values that was used in mapping the values of the membership function which was needed by the fuzzy logic system must be properly defined [11];

- All discrete variables with nominal values or Boolean (yes/no) are defined using the values: $0,1,2 \ldots \ldots$

- All continuous variables which are measured are expressed as a proportion of 1; i.e. $1.76 \%$ and $45 \%$ read as 0.176 and 0.45 separately into the suitable membership functions

The Table one (1) below make available a comprehensive explanation for the values of the labels to be used for each variable alongside with their respective membership functions. 
The International Journal of Computational Science, Information Technology and Control Engineering (IJCSITCE) Vol.5, No.2, April 2018

Table 1: Description of the labels for each variables

\begin{tabular}{|c|c|c|c|c|c|}
\hline No & Variable & \multicolumn{3}{|c|}{ Membership function mapping (label $=$ value $)$} & \multirow{2}{*}{\begin{tabular}{|c|}
$\begin{array}{l}\text { Membership } \\
\text { function }\end{array}$ \\
trapmf
\end{tabular}} \\
\hline 1 & $\begin{array}{l}\text { Blood } \\
\text { Urea Test }\end{array}$ & $\begin{array}{c}<20 \% \text { converts to } \\
\quad<0.2 \\
\rightarrow \mathrm{t}_{1}<0.2\end{array}$ & $\begin{array}{c}20 \% \leq \text { test } \leq 40 \% \text { converts } \\
\text { to } \\
0.2=<\text { test }<=0.4 \\
\rightarrow 0.2 \leq \mathrm{t}_{1} \leq 0.4\end{array}$ & $\begin{array}{c}>40 \% \text { converts } \\
\text { to }>0.4 \\
\rightarrow t_{1}>0.4\end{array}$ & \\
\hline 2 & eGFR & $\begin{array}{c}<0.9 \% \text { converts to } \\
\quad<0.09 \\
\rightarrow \mathrm{t}_{2}<0.09\end{array}$ & $\begin{array}{c}0.9 \% \leq \text { test } \leq 40 \% \text { converts } \\
\text { to } \\
0.2=<\text { test }<=0.4 \\
\rightarrow 0.2 \leq t_{2} \leq 0.4\end{array}$ & $\begin{array}{c}>40 \% \text { converts } \\
\text { to }>0.4 \\
\rightarrow t_{2}>0.4\end{array}$ & trapmf \\
\hline 3 & $\begin{array}{l}\text { Urea } \\
\text { Clearance } \\
\text { test }\end{array}$ & $\begin{array}{c}<6.4 \% \text { converts to } \\
\quad<0.64 \\
\rightarrow \mathrm{t}_{3}<6.4\end{array}$ & $\begin{array}{c}6.4 \% \leq \text { test } \leq 9.9 \% \text { converts } \\
\text { to } \\
0.64=<\text { test }<=0.99 \\
\rightarrow 0.64 \leq \mathrm{t}_{3} \leq 0.99\end{array}$ & $\begin{array}{c}>9.9 \% \text { converts } \\
\text { to }>0.99 \\
\rightarrow \mathrm{t}_{3}>0.99\end{array}$ & Trapmf \\
\hline 4 & $\begin{array}{l}\text { Creatinine } \\
\text { clearance } \\
\text { test }\end{array}$ & $\begin{array}{c}<0.93 \% \text { converts } \\
\text { to } \\
<0.093 \\
\rightarrow \mathrm{t}_{4}<0.2\end{array}$ & $\begin{array}{c}0.93 \% \leq \text { test } \leq 1.33 \% \\
\text { converts to } \\
0.093=<\text { test }<=0.133 \\
\rightarrow 0.093 \leq \mathrm{t}_{4} \leq 0.133\end{array}$ & $\begin{array}{c}>1.33 \% \\
\text { converts to } \\
>0.133 \\
\rightarrow \mathrm{t}_{4}>0.133\end{array}$ & Trapmf \\
\hline 5 & $\begin{array}{l}\text { Likelihood } \\
\text { of CKD }\end{array}$ & $\begin{array}{r}\mathrm{No} \\
\rightarrow \mathrm{X}=\end{array}$ & & $\begin{array}{l}\text { Yes } \\
x=1\end{array}$ & \\
\hline
\end{tabular}

\section{e. Fuzzification of the variables}

The triangular membership functions were used for the purpose of this study to map the degree of membership of the labels of each variable used in both input and output variable [12].Also, following the same pattern of description explained above, each of the under listed variable and the type of membership function used for the labels alongside the ordered pair that was used in mapping the degree of membership for each variable's label [12].

\section{a. Blood Urea Test, t1 (Figure 3)}

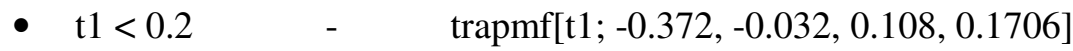

trapmf $[\mathrm{t} 1 ;-0.372,-0.032,0.108,0.1706]$

$$
=\left\{\begin{array}{cc}
0, & \mathrm{t} 1 \leq-0.372 \\
\frac{\mathrm{t} 1+0.372}{0.34} & -0.372 \leq \mathrm{t} 1 \leq-0.032 \\
1, & -0.372 \leq \mathrm{t} 1 \leq 0.108 \\
\frac{0.1706-\mathrm{t} 1}{0.108} & 0.108 \leq \mathrm{t} 1 \leq 0.1706 \\
0, & 0.1706 \leq \mathrm{t} 1
\end{array}\right\}
$$
- $\quad 0.2 \leq \mathrm{t} 1 \leq 0.4$
$-$
trapmf[t $1 ; 0.14520 .20420 .30520 .3602]$

trapmf[t1; 0.14520 .20420 .30520 .3602$]$ 
The International Journal of Computational Science, Information Technology and Control Engineering (IJCSITCE) Vol.5, No.2, April 2018

$$
=\left\{\begin{array}{cc}
0, & \mathrm{t} 1 \leq 0.1452 \\
\frac{\mathrm{t} 1-0.1452,}{0.059} & 0.1452 \leq \mathrm{t} 1 \leq 0.2042 \\
1, & 0.2042 \leq \mathrm{t} 1 \leq 0.3052 \\
\frac{0.3602-\mathrm{t} 1,}{0.055} & 0.3052 \leq \mathrm{t} 1 \leq 0.3602 \\
0, & 0.3602 \leq \mathrm{t} 1
\end{array}\right\}
$$

$\begin{array}{lllllll}- & \mathrm{t} 1>0.4 & - & \operatorname{trapmf}[\mathrm{t} 1 ; 0.343 & 0.3722 & 0.489 & 1.07]\end{array}$

trapmf [t1; 0.3430 .37220 .4891 .07$]$

$$
=\left\{\begin{array}{cc}
0, & \mathrm{t} 1 \leq 0.343 \\
\frac{\mathrm{t} 1-0.343}{0.0292}, & 0.343 \leq \mathrm{t} 1 \leq 0.372 \\
1, & 0.3722 \leq \mathrm{t} 1 \leq 0.3052 \\
\frac{1.07-\mathrm{t} 1,}{0.055} & 0.489 \leq \mathrm{t} 1 \leq 1.07 \\
0, & 1.07 \leq \mathrm{t} 1
\end{array}\right\}
$$

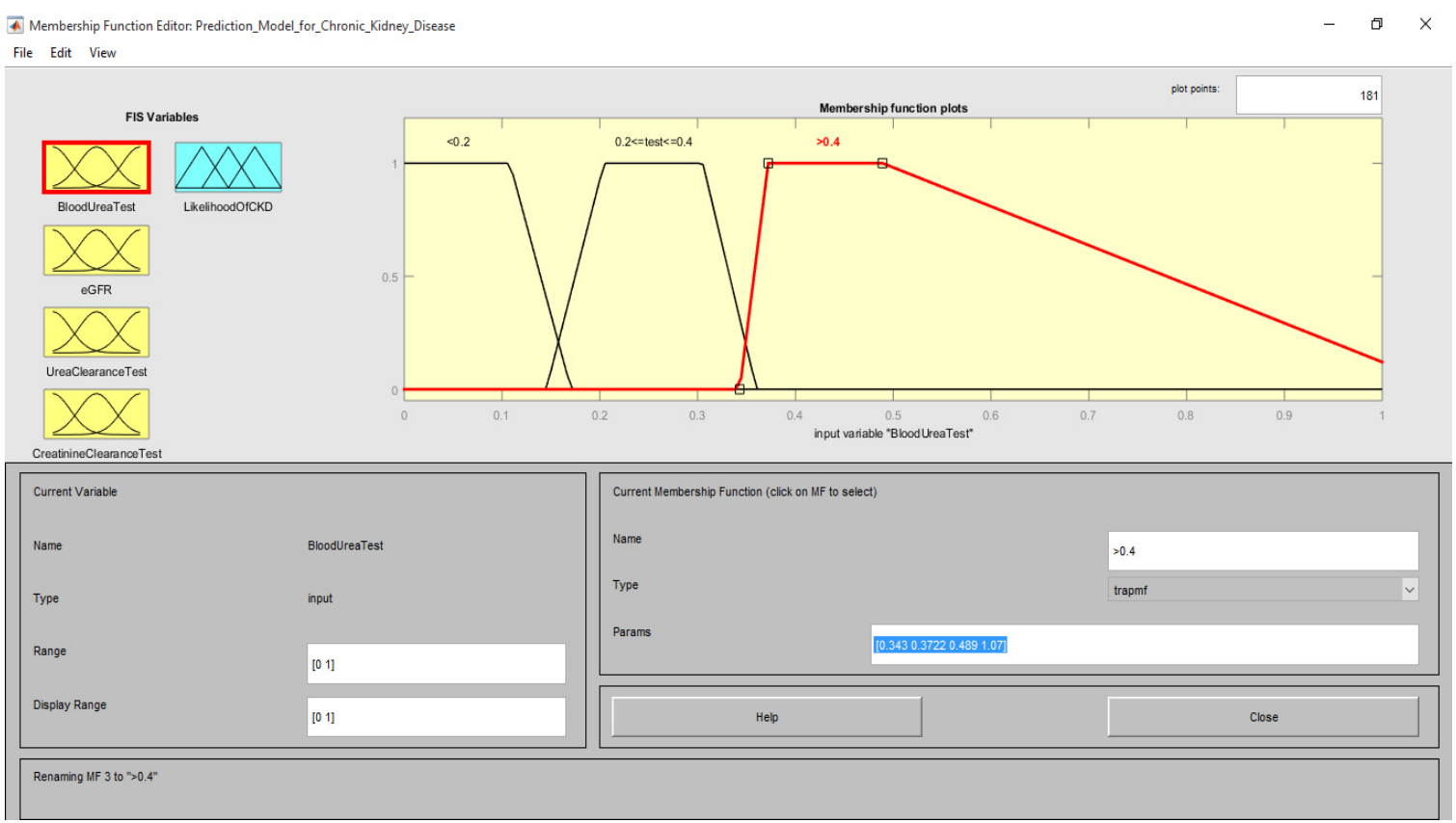

Figure 3: Membership function for Blood Urea Test 
The International Journal of Computational Science, Information Technology and Control Engineering (IJCSITCE) Vol.5, No.2, April 2018

b. eGFR, t2 (Figure 4)

- $\quad \mathrm{t} 2<0.09$

trapmf [-0.36, -0.04, 0.082880 .199$]$

trapmf [-0.36, -0.04, 0.082880 .199$]$

$$
=\left\{\begin{array}{cc}
0, & \mathrm{t} 2 \leq 0.36 \\
\frac{\mathrm{t} 2+0.36}{0.32}, & -0.36 \leq \mathrm{t} 2 \leq-0.04 \\
1, & -0.04 \leq \mathrm{t} 2 \leq 0.0829 \\
\frac{0.199-\mathrm{t} 2}{0.1161} & 0.0829 \leq \mathrm{t} 2 \leq 0.199 \\
0, & 0.199 \leq \mathrm{t} 2
\end{array}\right\}
$$

- $0.09 \leq \mathrm{t} 2<0.120$

trapmf [ $\left.\begin{array}{llll}0.171 & 0.225 & 0.276 & 0.3602\end{array}\right]$ trapmf [ [ $\left.\begin{array}{llll}0.171 & 0.225 & 0.276 & 0.3602\end{array}\right]$

$$
=\left\{\begin{array}{cc}
0, & \mathrm{t} 2 \leq 0.171 \\
\frac{\mathrm{t} 2-0.171}{0.054}, & 0.171 \leq \mathrm{t} 2 \leq 0.225 \\
1, & 0.225 \leq \mathrm{t} 2 \leq 0.276 \\
\frac{0.3602-\mathrm{t} 2}{0.0842} & 0.3602 \leq \mathrm{t} 2 \\
0, &
\end{array}\right\}
$$

- $\quad \mathrm{t} 2>0.120$ - $\quad$ trapmf [0.337 0.376 0.392 0.6647] trapmf[0.337 0.3760 .3920 .6647$]$

$$
=\left\{\begin{array}{cc}
0, & \mathrm{t} 2 \leq 0.337 \\
\frac{\mathrm{t} 2-0.337}{0.039}, & 0.337 \leq \mathrm{t} 2 \leq 0.376 \\
1, & 0.376 \leq \mathrm{t} 2 \leq 0.392 \\
\frac{0.6647-\mathrm{t} 2}{0.2727}, & 0.392 \leq \mathrm{t} 2 \leq 0.6647 \\
0, & 0.6647 \leq \mathrm{t} 2
\end{array}\right\}
$$


The International Journal of Computational Science, Information Technology and Control Engineering (IJCSITCE) Vol.5, No.2, April 2018

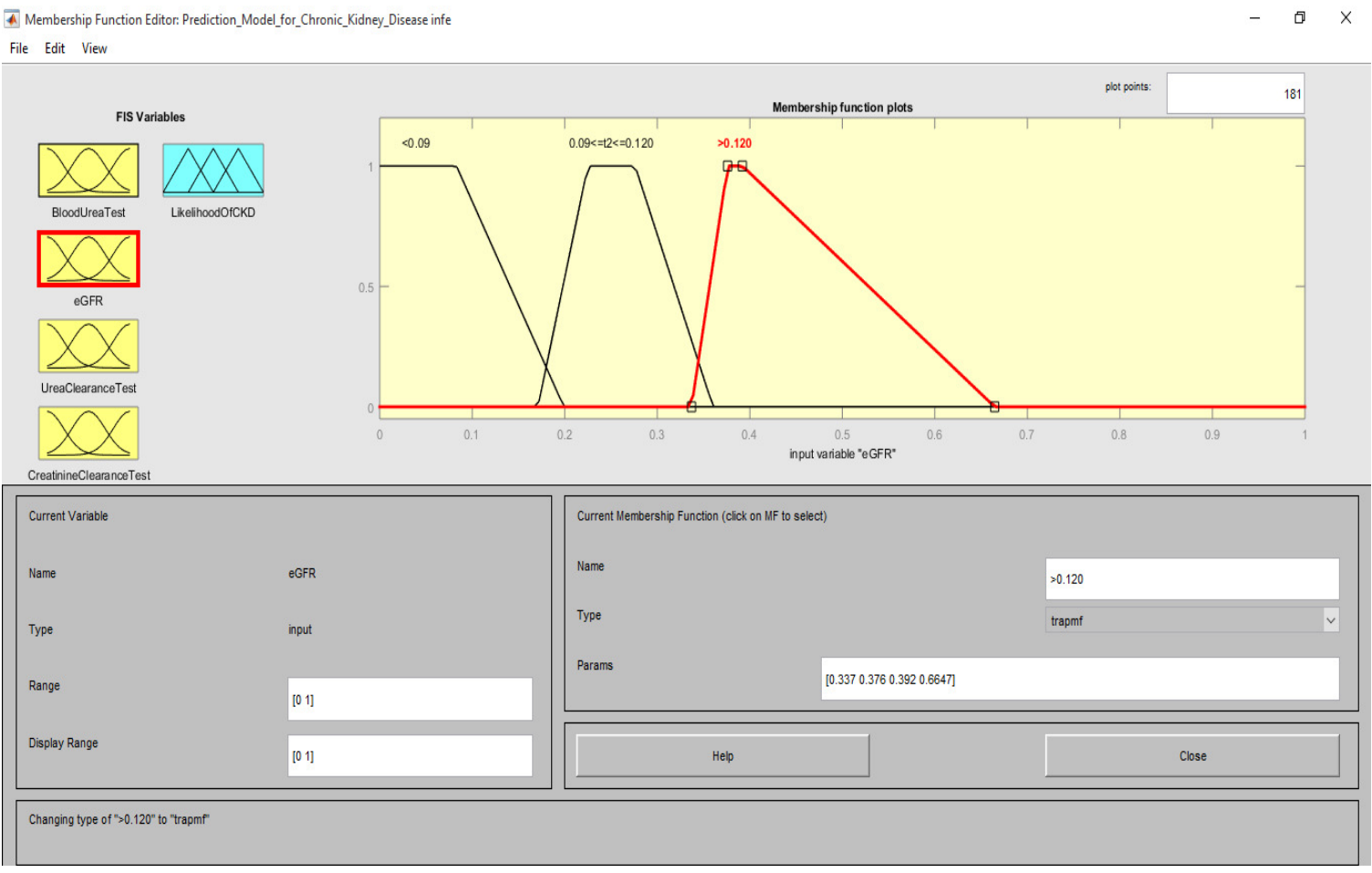

Figure 4: Membership function for eGFR

\section{c. Urea Clearance Test, (Figure 5)}

- $\mathrm{t} 3<0.64$ trapmf [-0.36 -0.04 0.08722 0.244]

trapmf [-0.36 -0.04 0.08722 0.244]

$$
=\left\{\begin{array}{cc}
0, & \mathrm{t} 3 \leq-0.36 \\
\frac{\mathrm{t} 3+0.36}{0.32}, & -0.36 \leq \mathrm{t} 3 \leq-0.04 \\
1, & -0.04 \leq \mathrm{t} 3 \leq 0.0872 \\
\frac{0.244-\mathrm{t} 3}{0.1568} & 0.0872 \leq \mathrm{t} 3 \leq 0.244 \\
0, & 0.244 \leq \mathrm{t} 3
\end{array}\right\}
$$

- $\quad 0.64 \leq \mathrm{t} 3 \leq 0.99-\quad \operatorname{trapmf}[0.19810 .26210 .46610 .8456]$ trapmf [ [ [ $\left.\begin{array}{llll}0.1981 & 0.2621 & 0.4661 & 0.8456\end{array}\right]$ 
The International Journal of Computational Science, Information Technology and Control Engineering (IJCSITCE) Vol.5, No.2, April 2018

$$
=\left\{\begin{array}{cc}
0, & \mathrm{t} 3 \leq 0.198 \\
\frac{\mathrm{t} 3-0.198}{0.064}, & 0.198 \leq \mathrm{t} 3 \leq 0.262 \\
1, & 0.262 \leq \mathrm{t} 3 \leq 0.466 \\
\frac{0.846-\mathrm{t} 3}{0.38} & 0.466 \leq \mathrm{t} 3 \leq 0.846 \\
0, & 0.846 \leq \mathrm{t} 3
\end{array}\right\}
$$

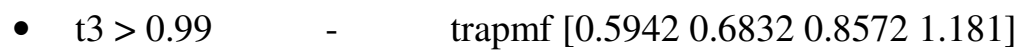

$$
\begin{aligned}
& \text { trapmf [ } \left.\begin{array}{llll}
0.5942 & 0.6832 & 0.8572 & 1.181
\end{array}\right] \\
& =\left\{\begin{array}{cc}
0, & \mathrm{t} 3 \leq 0.594 \\
\frac{\mathrm{t} 3-0.594}{0.089} & 0.594 \leq \mathrm{t} 3 \leq 0.683 \\
1, & 0.683 \leq \mathrm{t} 3 \leq 0.857 \\
\frac{1.181-\mathrm{t} 3}{0.324} & 0.857 \leq \mathrm{t} 3 \leq 1.181 \\
0, & 1.181 \leq \mathrm{t} 3
\end{array}\right\}
\end{aligned}
$$

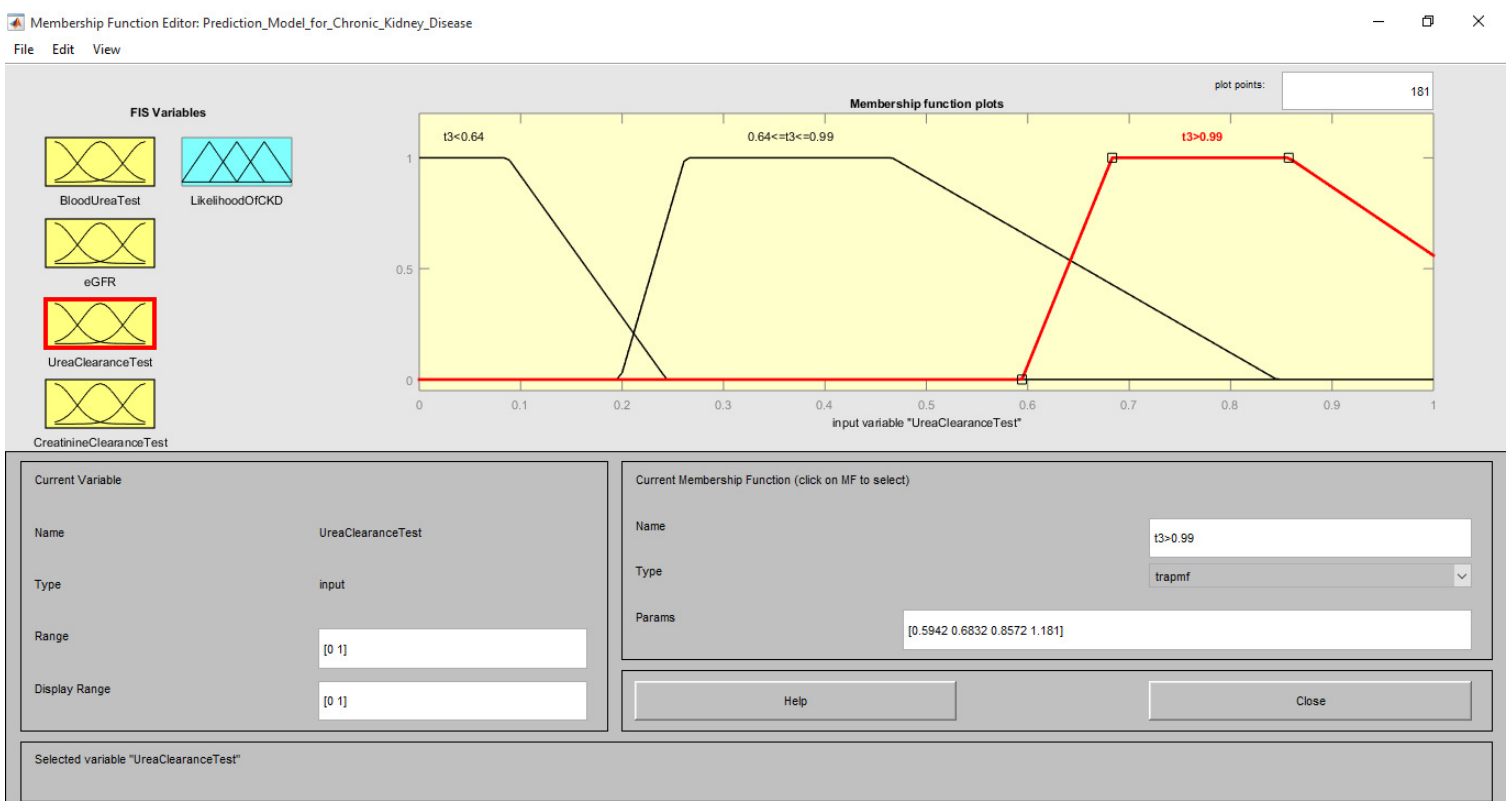

Figure 5: Membership function for Urea Clearance Test 
The International Journal of Computational Science, Information Technology and Control Engineering (IJCSITCE) Vol.5, No.2, April 2018

\section{d. Creatinine Clearance Test, t4 (Figure 6)}

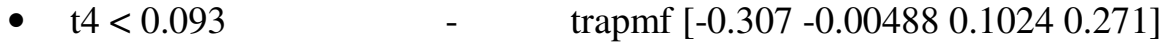
trapmf [-0.307-0.00488 0.1024 0.271]

$$
=\left\{\begin{array}{cc}
0, & \mathrm{t} 4 \leq-0.307 \\
\frac{\mathrm{t} 4+0.307,}{0.3021} & -0.307 \leq \mathrm{t} 4 \leq-0.0049 \\
1, & -0.0049 \leq \mathrm{t} 4 \leq 0.102 \\
\frac{0.271-\mathrm{t} 4}{0.169} & 0.102 \leq \mathrm{t} 4 \leq 0.271 \\
0, & 0.271 \leq \mathrm{t} 4
\end{array}\right\}
$$

- $\quad 0.093 \leq \mathrm{t} 4 \leq 0.133 \quad-\quad$ trapmf $[0.1940 .3180 .4880 .6538]$ trapmf [0.194 0.3180 .4880 .6538 ]

$$
=\left\{\begin{array}{cc}
0, & \mathrm{t} 4 \leq 0.194 \\
\frac{\mathrm{t} 4-0.194,}{0.124} & 0.194 \leq \mathrm{t} 4 \leq 0.318 \\
1, & 0.318 \leq \mathrm{t} 4 \leq 0.488 \\
\frac{0.654-\mathrm{t} 4}{0.166} & 0.488 \leq \mathrm{t} 4 \leq 0.654 \\
0, & 0.654 \leq \mathrm{t} 4
\end{array}\right\}
$$

- $\mathrm{t} 4>0.133$

trapmf [0.564 0.60620 .7211 .28 ] trapmf [0.564 0.6062 0.721 1.28]

$$
=\left\{\begin{array}{cc}
0, & \mathrm{t} 4 \leq 0.564 \\
\frac{\mathrm{t} 4-0.564,}{0.042} & 0.564 \leq \mathrm{t} 4 \leq 0.606 \\
1, & 0.606 \leq \mathrm{t} 4 \leq 0.721 \\
\frac{1.28-\mathrm{t} 4}{0.559} & 0.721 \leq \mathrm{t} 4 \leq 1.28 \\
0, & 1.28 \leq \mathrm{t} 4
\end{array}\right\}
$$


The International Journal of Computational Science, Information Technology and Control Engineering (IJCSITCE) Vol.5, No.2, April 2018

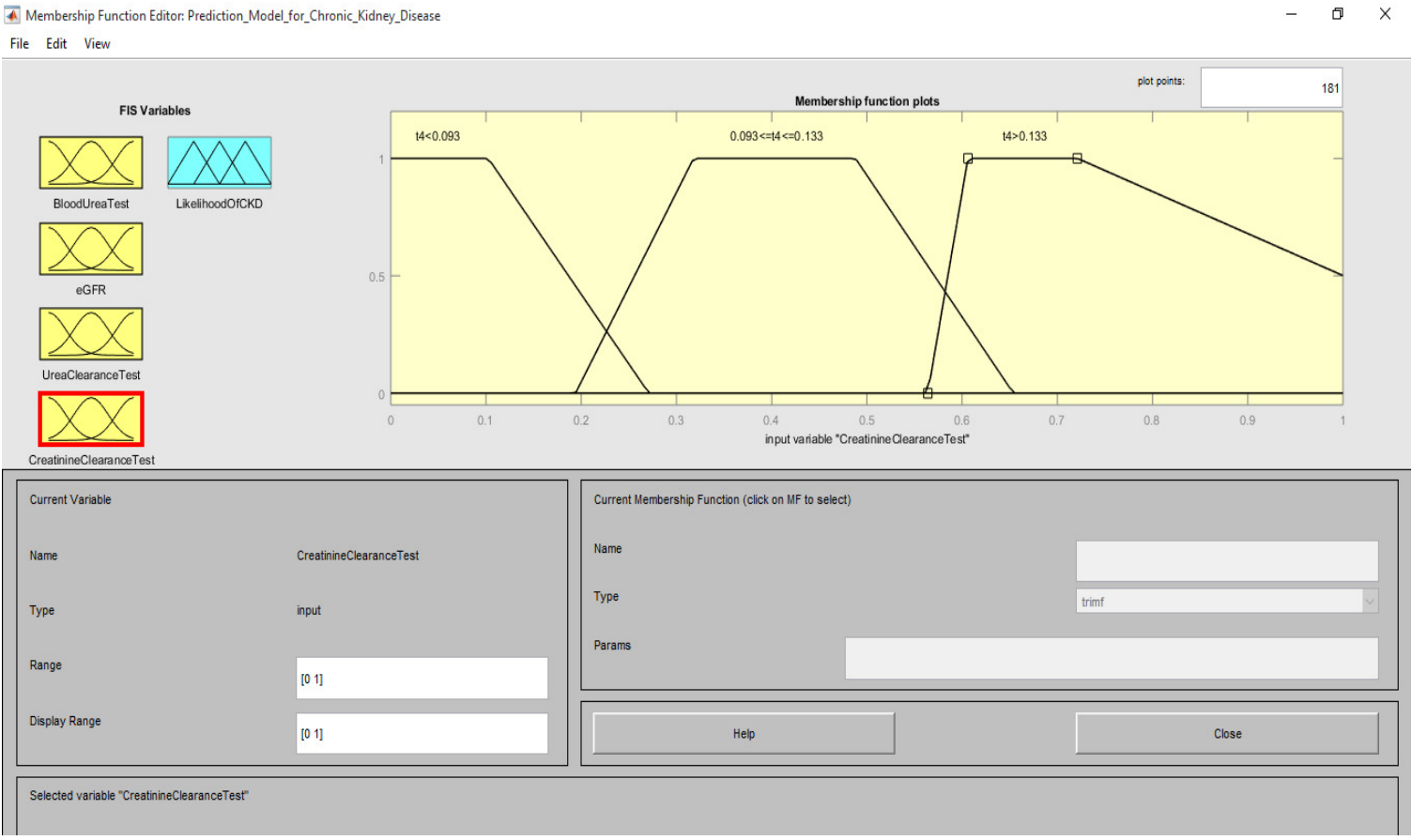

Figure 6: Membership function for Creatinine Clearance Test

\subsection{Inference engine development, aggregation and defuzzification}

For the proposed study in this paper, the first step was identifying the problems and then gathering of data and collection. Development of membership function followed and the next step is development of Inference engine, aggregation and defuzzification. For the development of the fuzzy inference engine for this paper, 32 different rules was formed as shown in Table 2 below. The fuzzy inference engine is used to formulate the mapping from a given input to an output using fuzzy logic [13]. The mapping then provides a basis from which decisions can be made, or patterns discerned. The 32 different rules serves as the input for the proposed model which are used to map each interval membership functions. After mapping each interval membership function, Case based reasoning is used to provide results. Cased based reasoning is the process of solving new problems based on the solutions of similar past problems or experience from an expert.

For the purpose of this paper, the "AND" and "OR" method is used for assessing each degree of membership. Adebayo et al (2015) critically explained this methods [14]:

The And Method is used in evaluating each degree of membership is Minimum (it selects the smallest value of many), the Or Method used is the Maximum (it selects the largest value out of many). For deriving the minimum the Implication Method is used which specifies how a fuzzy logic controller scales the membership functions of an output linguistic variable based on the rule weight of the corresponding rule. The rule weight is the truth value of the aggregated rule antecedent multiplied by the degree of support that one specify for the rule. This is done before defuzzification [14]. 
The International Journal of Computational Science, Information Technology and Control Engineering (IJCSITCE) Vol.5, No.2, April 2018

These fuzzy operators were used to determine the output for each rules which now necessitate aggregation to be functional in order to get a single output. The Aggregation method used in developing the prime output membership function is select to be Maximum (it selects the biggest value for all section of the output variable's membership function) [14]. This method was favoured since it is the most habitually used procedure of aggregating linear-wise membership functions like trapezoidal membership functions.

Fuzzy rules developed for the inference system

1. If (BloodUreaTest is $0.2<=$ test $<=0.4$ ) and (eGFR is $0.09<=\mathrm{t} 2<=0.120$ ) and (UreaClearanceTest is $0.64<=\mathrm{t} 3<=0.99$ ) and (CreatinineClearanceTest is $0.093<=\mathrm{t} 4<=0.133$ ) then (LikelihoodOfCKD is No) (1)

2. If (BloodUreaTest is <0.2) and (eGFR is $<0.09)$ and (UreaClearanceTest is $\mathrm{t} 3<0.64$ ) and (CreatinineClearanceTest is $t 4<0.093$ ) then (LikelihoodOfCKD is Yes) (1)

3. If (BloodUreaTest is $<0.2)$ and (eGFR is $<0.09$ ) and (UreaClearanceTest is $\mathrm{t} 3<0.64$ ) and (CreatinineClearanceTest is $0.093<=\mathrm{t} 4<=0.133$ ) then (LikelihoodOfCKD is Yes) (1)

4. If (BloodUreaTest is <0.2) and (eGFR is <0.09) and (UreaClearanceTest is $\mathrm{t} 3<0.64$ ) and (CreatinineClearanceTest is $t 4>0.133$ ) then (LikelihoodOfCKD is Yes) (1)

5. If (BloodUreaTest is <0.2) and (eGFR is <0.09) and (UreaClearanceTest is $0.64<=\mathrm{t} 3<=0.99$ ) and (CreatinineClearanceTest is $t 4<0.093$ ) then (LikelihoodOfCKD is Yes) (1)

6. If (BloodUreaTest is <0.2) and (eGFR is <0.09) and (UreaClearanceTest is $\mathrm{t} 3>0.99$ ) and (CreatinineClearanceTest is $t 4<0.093$ ) then (LikelihoodOfCKD is Yes) (1)

7. If (BloodUreaTest is $<0.2$ ) and (eGFR is $0.09<=\mathrm{t} 2<=0.120$ ) and (UreaClearanceTest is $\mathrm{t} 3<0.64)$ and (CreatinineClearanceTest is $\mathrm{t} 4<0.093$ ) then (LikelihoodOfCKD is Yes) (1)

8. If (BloodUreaTest is $<0.2$ ) and (eGFR is $>0.120$ ) and (UreaClearanceTest is $\mathrm{t} 3<0.64$ ) and (CreatinineClearanceTest is $t 4<0.093$ ) then (LikelihoodOfCKD is Yes) (1)

9. If (BloodUreaTest is $0.2<=$ test $<=0.4$ ) and (eGFR is $<0.09$ ) and (UreaClearanceTest is $\mathrm{t} 3<0.64)$ and (CreatinineClearanceTest is $\mathrm{t} 4<0.093$ ) then (LikelihoodOfCKD is Yes) (1)

10. If (BloodUreaTest is $>0.4$ ) and (eGFR is $<0.09$ ) and (UreaClearanceTest is $t 3<0.64$ ) and (CreatinineClearanceTest is $t 4<0.093$ ) then (LikelihoodOfCKD is Yes) (1)

11. If (BloodUreaTest is $<0.2$ ) or (eGFR is $<0.09$ ) or (UreaClearanceTest is $t 3<0.64$ ) or (CreatinineClearanceTest is $t 4<0.093$ ) then (LikelihoodOfCKD is Yes) (1)

12. If (BloodUreaTest is $<0.2$ ) or (eGFR is $<0.09$ ) or (UreaClearanceTest is $t 3<0.64$ ) or (CreatinineClearanceTest is $0.093<=\mathrm{t} 4<=0.133$ ) then (LikelihoodOfCKD is Yes) (1)

13. If (BloodUreaTest is $<0.2$ ) or (eGFR is $<0.09$ ) or (UreaClearanceTest is $t 3<0.64$ ) or (CreatinineClearanceTest is $t 4>0.133$ ) then (LikelihoodOfCKD is Yes) (1) 
The International Journal of Computational Science, Information Technology and Control Engineering (IJCSITCE) Vol.5, No.2, April 2018

14. If (BloodUreaTest is $<0.2$ ) or (eGFR is $<0.09$ ) or (UreaClearanceTest is $0.64<=t 3<=0.99$ ) or (CreatinineClearanceTest is $t 4<0.093$ ) then (LikelihoodOfCKD is Yes) (1)

15. If (BloodUreaTest is $<0.2$ ) or (eGFR is $<0.09$ ) or (UreaClearanceTest is $t 3>0.99$ ) or (CreatinineClearanceTest is $t 4<0.093$ ) then (LikelihoodOfCKD is Yes) (1)

16. If (BloodUreaTest is $<0.2$ ) or (eGFR is $0.09<=\mathrm{t} 2<=0.120$ ) or (UreaClearanceTest is $\mathrm{t} 3<0.64)$ or (CreatinineClearanceTest is $\mathrm{t} 4<0.093$ ) then (LikelihoodOfCKD is Yes) (1)

17. If (BloodUreaTest is $<0.2$ ) or (eGFR is $>0.120$ ) or (UreaClearanceTest is $t 3<0.64$ ) or (CreatinineClearanceTest is $t 4<0.093$ ) then (LikelihoodOfCKD is Yes) (1)

18. If (BloodUreaTest is $0.2<=$ test $<=0.4$ ) or (eGFR is $<0.09$ ) or (UreaClearanceTest is $\mathrm{t} 3<0.64)$ or $($ CreatinineClearanceTest is $\mathrm{t} 4<0.093$ ) then (LikelihoodOfCKD is Yes) (1)

19. If (BloodUreaTest is $>0.4$ ) or (eGFR is $<0.09$ ) or (UreaClearanceTest is $t 3<0.64$ ) or (CreatinineClearanceTest is $t 4<0.093$ ) then (LikelihoodOfCKD is Yes) (1)

20. If (BloodUreaTest is $<0.2$ ) or (eGFR is $<0.09$ ) or (UreaClearanceTest is $t 3<0.64$ ) or (CreatinineClearanceTest is $0.093<=\mathrm{t} 4<=0.133$ ) then (LikelihoodOfCKD is Yes) (1)

21. If (BloodUreaTest is $<0.2$ ) or (eGFR is $<0.09$ ) or (UreaClearanceTest is $\mathrm{t} 3<0.64$ ) or (CreatinineClearanceTest is $t 4>0.133$ ) then (LikelihoodOfCKD is Yes) (1)

22. If (BloodUreaTest is $<0.2$ ) or (eGFR is $<0.09$ ) or (UreaClearanceTest is $0.64<=t 3<=0.99$ ) or (CreatinineClearanceTest is $t 4<0.093$ ) then (LikelihoodOfCKD is Yes) (1)

23. If (BloodUreaTest is $<0.2$ ) or (eGFR is $<0.09$ ) or (UreaClearanceTest is $0.64<=\mathrm{t} 3<=0.99$ ) or (CreatinineClearanceTest is $t 4>0.133$ ) then (LikelihoodOfCKD is Yes) (1)

24. If (BloodUreaTest is <0.2) and (eGFR is <0.09) and (UreaClearanceTest is $0.64<=\mathrm{t} 3<=0.99$ ) and (CreatinineClearanceTest is $\mathrm{t} 4<0.093$ ) then (LikelihoodOfCKD is Yes) (1)

25. If (BloodUreaTest is $<0.2$ ) and (eGFR is $<0.09$ ) and (UreaClearanceTest is $0.64<=\mathrm{t} 3<=0.99)$ and (CreatinineClearanceTest is $t 4>0.133)$ then (LikelihoodOfCKD is Yes) (1)

26. If (BloodUreaTest is $<0.2)$ and (eGFR is $0.09<=\mathrm{t} 2<=0.120)$ and (UreaClearanceTest is $0.64<=\mathrm{t} 3<=0.99)$ and (CreatinineClearanceTest is $\mathrm{t} 4>0.133$ ) then (LikelihoodOfCKD is Yes) (1)

27. If (BloodUreaTest is $<0.2)$ and (eGFR is $0.09<=\mathrm{t} 2<=0.120$ ) and (UreaClearanceTest is $0.64<=\mathrm{t} 3<=0.99)$ and $($ CreatinineClearanceTest is $\mathrm{t} 4<0.093)$ then (LikelihoodOfCKD is Yes) (1)

28. If (BloodUreaTest is $<0.2$ ) or (eGFR is $0.09<=\mathrm{t} 2<=0.120$ ) or (UreaClearanceTest is $0.64<=\mathrm{t} 3<=0.99$ ) or (CreatinineClearanceTest is $\mathrm{t} 4>0.133$ ) then (LikelihoodOfCKD is Yes) (1) 
The International Journal of Computational Science, Information Technology and Control Engineering (IJCSITCE) Vol.5, No.2, April 2018

29. If (BloodUreaTest is $<0.2$ ) or (eGFR is $0.09<=\mathrm{t} 2<=0.120$ ) or (UreaClearanceTest is $0.64<=\mathrm{t} 3<=0.99) \quad$ or $\quad$ (CreatinineClearanceTest is $0.093<=\mathrm{t} 4<=0.133$ ) then (LikelihoodOfCKD is Yes) (1)

30. If (BloodUreaTest is $0.2<=$ test $<=0.4$ ) or $\quad(\mathrm{eGFR}$ is $0.09<=\mathrm{t} 2<=0.120$ ) or (UreaClearanceTest is $0.64<=\mathrm{t} 3<=0.99$ ) or (CreatinineClearanceTest is $0.093<=\mathrm{t} 4<=0.133$ ) then (LikelihoodOfCKD is Yes) (1)

31. If (BloodUreaTest is $0.2<=$ test $<=0.4$ ) or (eGFR is $>0.120$ ) or (UreaClearanceTest is $0.64<=\mathrm{t} 3<=0.99)$ or $\quad$ (CreatinineClearanceTest is $0.093<=\mathrm{t} 4<=0.133$ ) then (LikelihoodOfCKD is Yes) (1)

32. If (BloodUreaTest is $0.2<=$ test $<=0.4$ ) or (eGFR is $>0.120$ ) or (UreaClearanceTest is t $3>0.99$ ) or (CreatinineClearanceTest is $0.093<=t 4<=0.133$ ) then (LikelihoodOfCKD is Yes) (1)

The defuzzification of the output membership function follow-on from the growth of fuzzy rules demonstrate that likelihood of CKD is NO only if (BloodUreaTest is $0.2<=$ test $<=0.4$ ) or (eGFR is $0.09<=\mathrm{t} 2<=0.120$ ) or (UreaClearanceTest is $0.64<=\mathrm{t} 3<=0.99$ ) or (CreatinineClearanceTest is $0.093<=t 4<=0.133)$. Figure 7 displays the diagram of the simulated fuzzy logic system for the prediction of the likelihood ofChronic Kidney Disease applicable for an individual given 4 input variables; blood urea test, eGFR (estimated glomerular filtration rate), Urea clearance test and Creatinine clearance test. Using MATLAB 2015a, a fuzzy inference system is developed using fuzzy logic toolbox. The simulated fuzzy logic system is of the Mamdani types and consist of 4 fuzzifed inputs and a single fuzzified outputs variables. Also an inference engine is developed using 32 IF-THEN rules relating expert knowledge - collected via knowledge elicitation from expert.

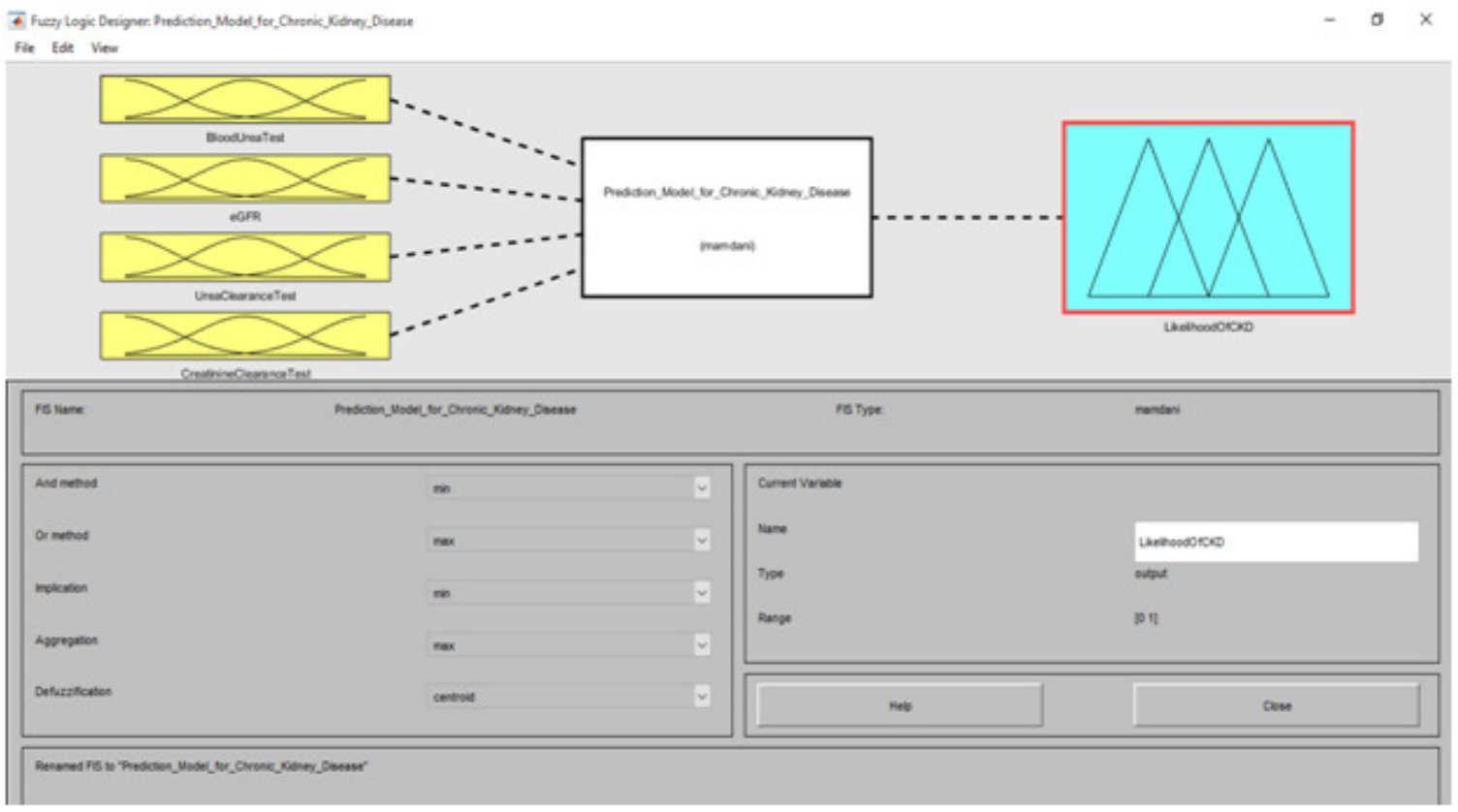

Figure 7: Proposed fuzzy inference system 
The International Journal of Computational Science, Information Technology and Control Engineering (IJCSITCE) Vol.5, No.2, April 2018

\section{RESULTS AND DISCUSSIONS}

The fuzzy logic inference system was used in modelling. The modelling tool used was MATLAB 2015a formulated. The fuzzy logic toolbox available on MATLAB is used for analysing, designing and simulating the predictive model using trapezoidal membership functions for the fuzzification of the input and output variables. The fuzzy logic system was used to achieve an assessment of the surface diagram which demonstrate the spreading of the numerous likely values and the association among any two variables.

Figure 8 offers a scheme of the surface diagram presenting the connection among blood urea test and eGFR test. From the diagram it is observed that cases of individuals having between $20 \%$ $40 \%$ level of blood urea test will likely not have CKD while cases above $40 \%$ and also less than $20 \%$ after blood urea test will likely have CKD. Also, the surface diagram provides information about the eGFR where cases between $90 \%$ - 120\% will likely not have CKD while cases above $120 \%$ and cases below $90 \%$ will likely have CKD;

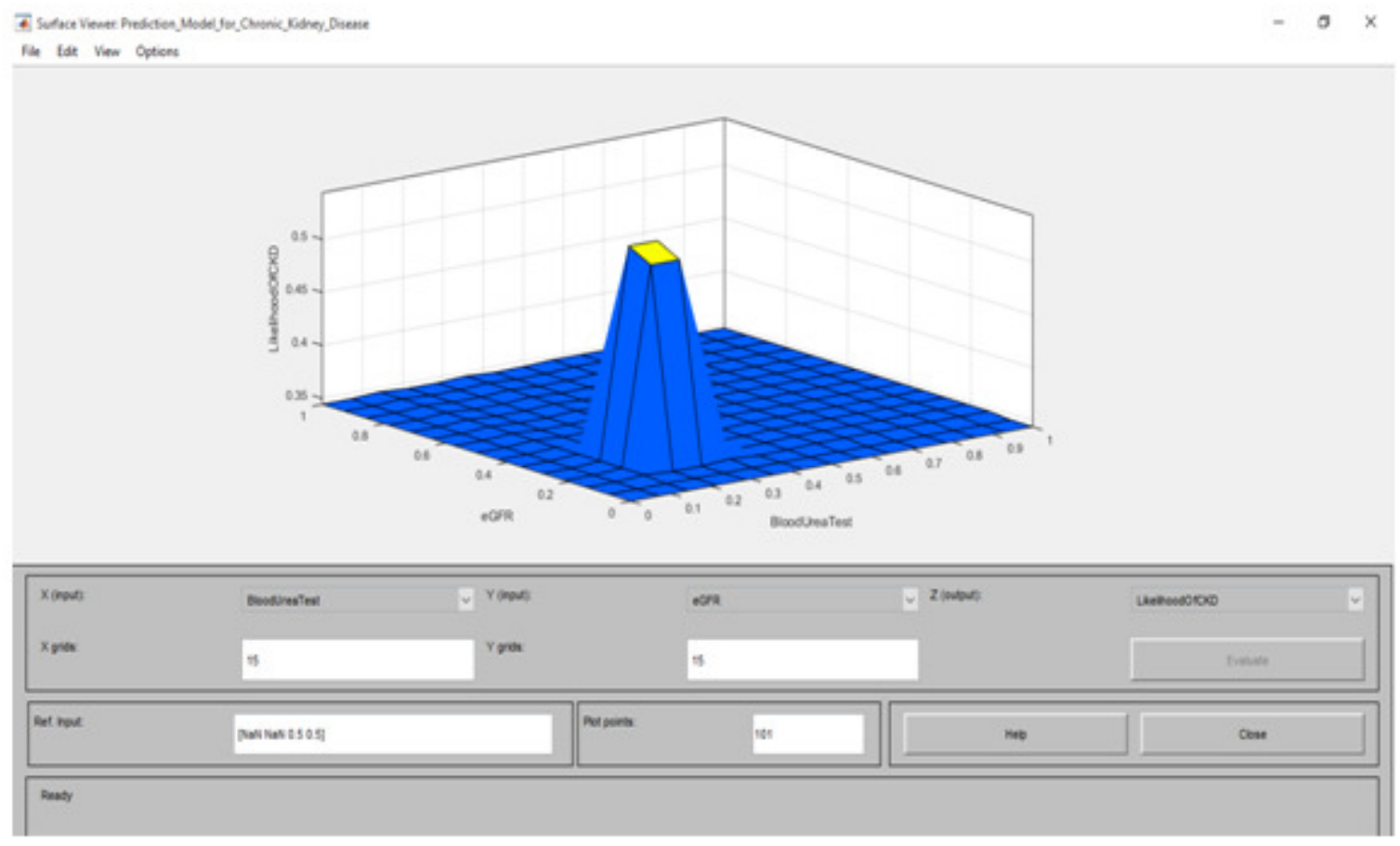

Figure 8: Surface diagram of Blood Urea test and eGFR

Figure 9 displays the surface diagram having a scheme of the connection among the eGFR test and Urea Clearance test. It can be observed that likelihood of CKD for eGFR occurs when test level is less than $90 \%$ and greater than $120 \%$. No likelihood of having CKD is observed for eGFR between values $90 \%$ to $120 \%$ and level $64 \%$ to $99 \%$ for Urea Clearance test.

Figure 10 illustrates the surface diagram of the bond that exists between the data values of Urea Clearance Test and Creatinine Clearance test. From the diagram, it is observed that likelihood of CKD occurs when Urea Clearance test is less than $64 \%$ or greater than $99 \%$. Also, for Creatinine Clearance, likelihood of CKD will occur when value data is less than $93 \%$ or greater than $133 \%$. 
The International Journal of Computational Science, Information Technology and Control Engineering (IJCSITCE) Vol.5, No.2, April 2018

No likelihood of CKD occurs for Urea Clearance test between 64\% to $99 \%$ and $93 \%$ to $133 \%$ for Creatinine Clearance test.

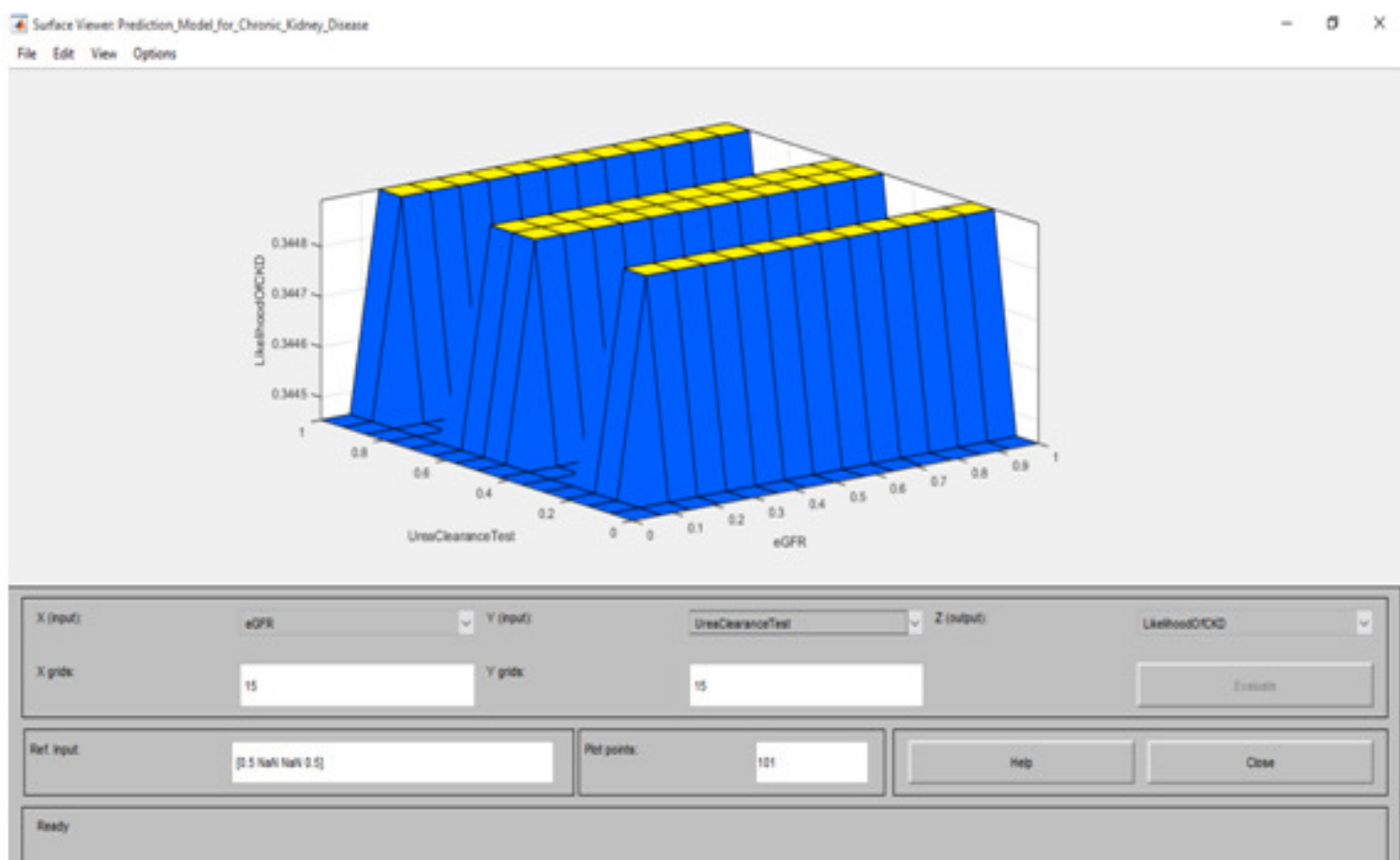

Figure 9: Surface diagram of eGFR and Urea Clearance test

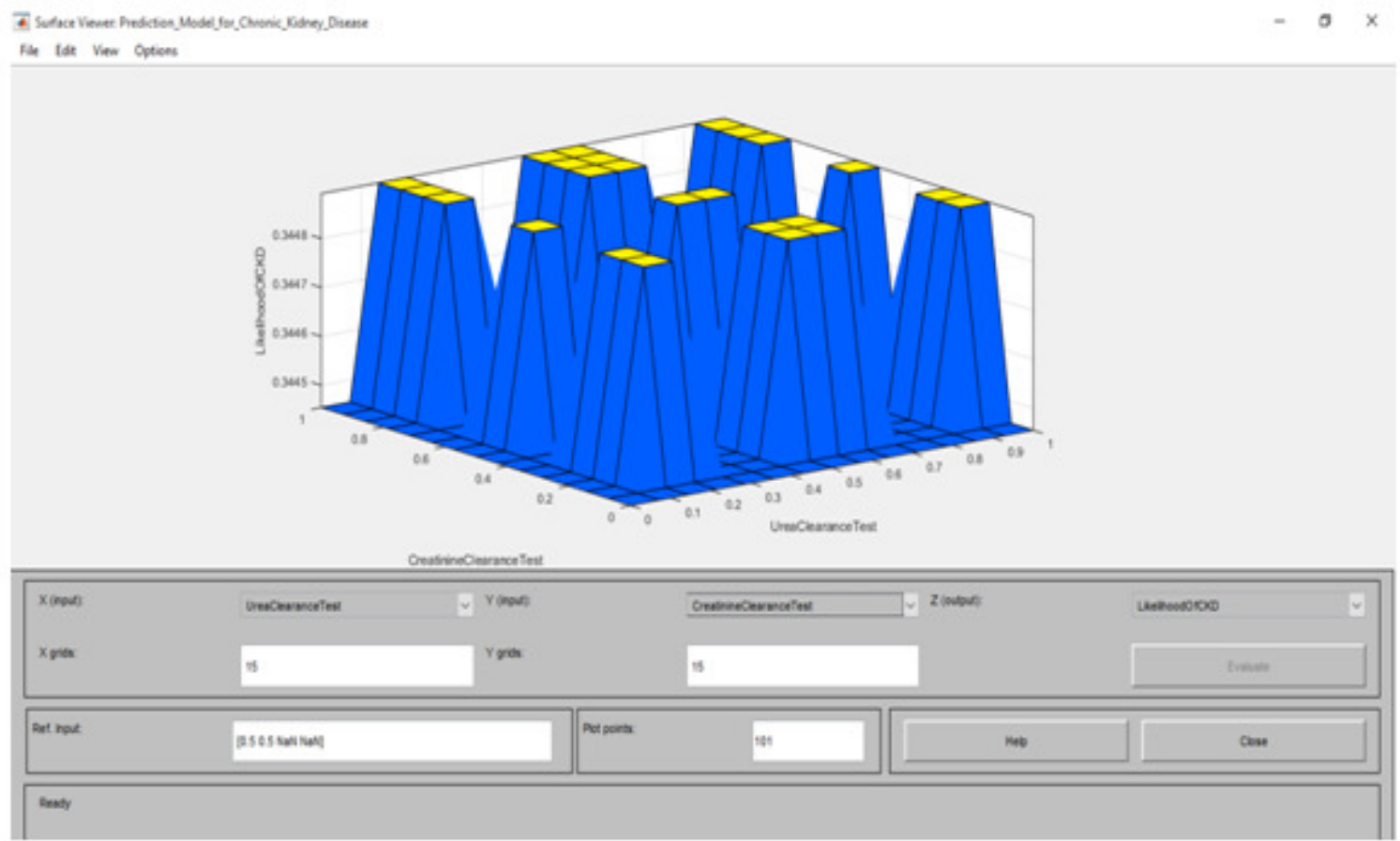

Figure 10: Surface diagram of Urea Clearance test and Creatinine Clearance test 
The International Journal of Computational Science, Information Technology and Control Engineering (IJCSITCE) Vol.5, No.2, April 2018

\section{CONCLUSION}

The model presented for the prediction of the likelihood of CKD was untaken using 4 input variables which are: blood urea test, eGFR (estimated glomerular filtration rate), Urea clearance test and Creatinine clearance test done on a patient. Each of the variables was properly identified and the relationship between them was well defined which was used in developing the fuzzy inference system. All variables were then fuzzified and the fuzzified input was used in developing the inference engine. The 32 output rule were produced which was the fuzzified to get crisp output (Yes or No). The presented model was computer-generated by the fuzzy logic toolbox available in MATLAB 2015a software and the results of the performance of the proposed model obtainable via the surface diagram. It is thought that this model can be used to carefully ascertain the likelihood of Chronic Kidney Disease among patients in a medical centre and hence provide necessary treatments.

\section{REFERENCES}

[1] National Kidney Foundation, (2017): A to Z health Guide: About Chronic Kidney Disease

[2] ChijiokeIremeka (2017) News Telegraph: Why more Nigerians will die of kidney diseases Nephrologists

(https://newtelegraphonline.com/2017/12/nigeriansll-die-kidney-diseases-nephrologists/).

[3] ChiomaObinna (2016). Vanguard Newspaper, Nigeria, Article Name: 17,000 kidney failure cases diagnosed annually in Nigeria (https://www.vanguardngr.com/2016/03/17000-kidney-failure-casesdiagnosed-annually-in-nigeria/)

[4] Oluseyi et al (2016). Chronic kidney disease in Nigeria: Late presentation is still the norm. Volume 57, Issue 3, Page : 185-189.

[5] Adebayo et al (2015). Predictive Model for Likelihood of Survival of Sickle-Cell Anaemia (SCA) among Paediatric Patients using Fuzzy Logic, Pg 32.

[6] Dr. Yusuf Perwej (2015). An Optimal Approach to Edge Detection Using Fuzzy Rule and Sobel Method: International Journal of Advanced Research in Electrical, Electronics and Instrumentation Engineering Pg. 8. Dept. of Computer Science \& Engineering, Al Baha University, Al Baha, Kingdom of Saudi, Arabia (KSA).

[7] Fuzzy Logic Toolbox User's Guide: MATLAB R2013a Pg 8.

[8] FarzadFirouziJahantigh (2015). Kidney Diseases Diagnosis by Using Fuzzy Logic, Department of Industrial Engineering, Faculty of Engineering, University of Sistan and Baluchestan, zahedan, Iran International Conference on Industrial Engineering and Operations Management Dubai, United Arab Emirates (UAE).

[9] KerinaBlessmoreChimwayi, NoorieHaris and Ronnie D. Caytiles (2017). Risk Level Prediction of Chronic Kidney Disease Using Neuro-Fuzzy and Hierarchical Clustering Algorithm (s), International Journal of Multimedia and Ubiquitous Engineering School of Computer Science and Engineering, VIT University.

[10] Huong et al (2017). A simple questionnaire to detect chronic kidney disease patients from Long An province screening data in Vietnam: BMC Research Notes 
The International Journal of Computational Science, Information Technology and Control Engineering

(IJCSITCE) Vol.5, No.2, April 2018

[11] Adebayo et al (2015). Predictive Model for Likelihood of Survival of Sickle-Cell Anaemia (SCA) among Paediatric Patients using Fuzzy Logic, Pg 35

[12] Adebayo et al (2015). Predictive Model for Likelihood of Survival of Sickle-Cell Anaemia (SCA) among Paediatric Patients using Fuzzy Logic, Pg 36

[13] Moses E. Ekpenyong, Udoinyang G. Inyang and EmemObong O. Udoh (2016). Adaptive Prosody Modelling for Improved Synthetic Speech Quality. Department of Computer Science, University of Uyo, Uyo, Nigeria 2016. Pg. 23.

[14] Adebayo et al (2015). Predictive Model for Likelihood of Survival of Sickle-Cell Anaemia (SCA) among Paediatric Patients using Fuzzy Logic, Pg 40.

\section{AUTHORS}

Ajinaja Micheal Olalekan is Computer Science graduate who earned his Bachelor Degree from ObafemiAwolowo University, Ile-Ife, Osun State. He developed the Computer Based Testing Application used by educational institutions in some part of the country. He has publication in IJCST on using Component Based Model in developing Software. He currently runs his Master's Degree program at University of Ibadan with focus on Predictive Model and Bioinformatics.

Ajinaja Olayinka David grew up at Ibadan, Southern Side of Nigeria. He earned a Bachelor Degree in Electronics and Electrical Engineering at ObafemiAwolowo University, Ile-Ife Osun state. He has interned with various companies which includes the department of Electrical and works, Ministry of Oyo State; Vodacom Nigeria and Cadbury Nigeria where he received a certificate of performance. He is CISCO certified Engineer and also has interest in fuzzy logic of controllers. $\mathrm{He}$ presently interns at Huawei Nigeria
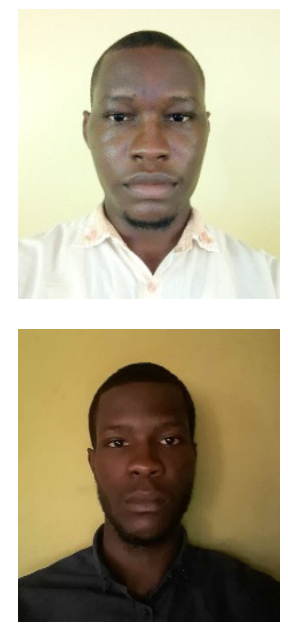